\title{
O Acolhimento dos Portadores de Sofrimento Mental da Estratégia Saúde da Família
}

\author{
The user embracement of pelople with suffering mental in health family \\ strategy
}

\author{
Eduardo Nogueira Cortez ${ }^{1}$, Edilene Aparecida Araujo da Silveira ${ }^{2}$, Mariana Moreira \\ Silva ${ }^{1}$, Paula Silva Alves ${ }^{1}$.
}

${ }^{1}$ Universidade Estadual de Minas Gerais - Campus Divinópolis, Divinópolis, Minas Gerais, Brasil.

${ }^{2}$ Universidade Federal de São João del Rei - Campus Centro Oeste Dona Lindu. Divinópolis-Minas Gerais, Brasil.

\section{Resumo}

Introdução: Os portadores de sofrimento mental são vistos nos serviços de saúde como pessoas difíceis de lidar e perigosas. Isso influencia o seu acolhimento pela atenção primária. Objetivo: Conhecer os sentidos presentes nas práticas discursivas dos enfermeiros de Estratégia Saúde da Família relacionadas ao acolhimento de pessoas em sofrimento mental. Metodologia: O estudo é de natureza qualitativa e usou o construcionismo social como perspectiva teórica. A coleta de dados foi realizada, por meio de entrevistas semi estruturadas, com 13 enfermeiros de diferentes ESF. Resultados: Após análise dos dados, identificou-se três categorias: Refletindo sobre o papel da atenção primária na assistência ao portador de sofrimento mental; acolhendo o portador de sofrimento mental; as dificuldades na prática do acolhimento. Conclusão: $\mathrm{O}$ relacionamento entre profissionais e usuários durante o acolhimento é marcado pela coexistência dos dois modelos assistenciais. Nesse embate, as práticas discursivas e as produções de sentido irão fortalecer um ou outro modelo e poderão trazer modificações em conteúdos relacionados ao cuidado do sofrimento mental em nossa sociedade.

Palavras-Chave: Acolhimento; Saúde mental; Relações enfermeiro-paciente

Autor correspondente:

Edilene Aparecida Araujo da Silveira

Endereço: R. Afranio Peixoto, 2211, Apto 203 Bloco C,

Recebido em: 13/07/2016

Divinópolis, $M G$ - Brasil

Telefone: +55 37 99192-5314

Revisado em: 26/09/2016

Aceito em: 29/09/2016

E-mail: edileneap@ufsj.edu.br

Publicado em: 07/12/2016 


\section{Abstract}

Introduction: The mental patients have been see in health services as people difficult to relationship and dangerous. This influences your reception for primary care. Objective: To know the meanings present in the discursive practices nurses of the Health Family Strategy related to User Embracement of people suffering mental problems. Methodology: The study is qualitative and used the social constructionism like a theoretical perspective. Data collection was conducted through semi-structured interviews with 13 nurses from different Health Family Estrategy. Results: After analyzing the data, we identified three categories: Reflecting on the role of primary care in the care of mental suffering; welcoming the mental suffering; difficulties in practice the host. Conclusion: The relationship between professionals and users during the host is marked by the coexistence of the two care models. In this clash, the discursive practices and sense of productions will strengthen one or another model and may bring changes in content related to the care of mental suffering in our society.

\section{Keywords: User Embracement; Mental health; nurse-patient relationships}

\section{Introdução}

Os estudos epidemiológicos realizados no Brasil acerca dos casos de transtornos mentais que demandam algum tipo de assistência médica apontam estimativas de $20 \%$, ou seja, a cada cinco brasileiros, um apresenta demanda para a saúde mental ${ }^{1}$. Apesar da alta prevalência de sofrimento mental, essa procura passa despercebida pelos serviços de saúde da atenção primária ${ }^{2}$.

A Organização Mundial da Saúde (OMS) e o Ministério da Saúde estimam que aproximadamente $80 \%$ das pessoas encaminhadas para os profissionais de saúde mental não trazem, a priori, uma demanda específica que justifique a necessidade de atenção especializada. Essa demanda deveria, portanto, ser acolhida pela Atenção Primária à Saúde ${ }^{3,4}$.

A Atenção Primária à Saúde e a Estratégia Saúde da Família (ESF) são as portas de entrada para o sistema de saúde, que recebem os usuários e particularmente, as pessoas que apresentam demandas de saúde mental. Nesses serviços de saúde, o acolhimento é o primeiro passo do atendimento na direção de assistência à saúde com qualidade. Este é um recurso terapêutico que resgata a dignidade humana, qualifica a atenção, confere maior competência e sensibilidade no reconhecimento de necessidades e demandas dos pacientes e comunidades $^{2}$.

O acolhimento proporciona o encontro entre usuário e profissional no relacionamento interpessoal e formação de vínculo, desencadeia a escuta e a empatia, se constitui em possibilidade de universalização do acesso e qualificação das relações ${ }^{2,5}$. Apesar de sua importância, o acolhimento enfrenta problemas em sua efetivação na área de saúde mental na Atenção Primária à Saúde. Dentre os obstáculos estão tendências ao encaminhamento, ausência de educação continuada e dúvidas quanto à implementação.
A dificuldade em acolher a pessoa com sofrimento mental pode ser identificada em vários municípios e este problema está presente mesmo quando a queixa se refere à saúde física e possui protocolos de atendimento definidos ${ }^{6}$. Além disso, uma revisão da literatura acerca do assunto apontou que há falta de uniformidade na prática do acolhimento, que pode ser trabalhado pelos profissionais de forma desarticulada e pontual ou de forma integral, longitudinal e interprofissional ${ }^{7}$.

A escolha por um modelo ou outro depende do significado que o acolhimento tem para a equipe de saúde e isso pode repercutir em sucessos e dificuldades percebidas por profissionais e usuários. Apesar de todos os profissionais estarem aptos para realizarem tal ação, o enfermeiro é aquele que mais frequentemente a executa. Sendo assim, este estudo buscou elucidar a questão: qual é o significado do acolhimento dos portadores de sofrimento mental nas ESF por enfermeiros em um município de médio porte do Centro Oeste de Minas Gerais?

Conforme o significado que o acolhimento assume para a equipe e o enfermeiro, os benefícios e a relevância do acolhimento podem se perder diante dos obstáculos enfrentados na prática ${ }^{7}$. Portanto, este estudo é relevante por conhecer os significados que os enfermeiros atribuem ao acolhimento. Ao identificá-los, podem ser elaboradas formas de intervenção em benefício de um acolhimento integrado às ações em saúde que considere a integralidade do indivíduo com sofrimento mental.

Destarte, foi objetivo do estudo conhecer os significados presentes nas práticas discursivas dos enfermeiros de ESF relacionadas ao acolhimento de pessoas em sofrimento mental. 


\section{Metodologia}

O estudo é de natureza qualitativa e usou o construcionismo social como perspectiva teórica. $\mathrm{O}$ construcionismo social é uma das abordagens sociológicas da psicologia social e recebe influências da etnometodologia, da sociologia, do feminismo e do pós-estruturalismo ${ }^{8}$.

Os acontecimentos, sob a ótica do construcionismo, recebem influência sócio-histórica e circulam em inúmeras versões na sociedade. $\mathrm{O}$ foco de interesse é a produção de sentidos nas práticas cotidianas, a construção da realidade pelas pessoas e a linguagem enquanto construtora de realidades e prática social ${ }^{8}$. Conforme o sentido atribuído ao acolhimento e aos indivíduos em sofrimento mental, o enfermeiro constrói a realidade contextualizada histórica e socialmente.

Na coleta de dados, foram utilizadas entrevistas semiestruturadas. Durante a entrevista, o participante se posiciona diante do assunto considerando a história sociocultural, pessoal e a relação com os outros sujeitos. Ele, então, representa sua experiência contextualizada, subjetiva e socialmente construída, por meio da linguagem falada e/ou escrita. Esse discurso pode estender-se do passado infinito ao futuro infinito, utilizando, inclusive, falas de pessoas que não estão presentes.

A entrevista individual foi realizada com 13 enfermeiros de ESF de um município de médio porte do Centro Oeste de Minas Gerais, no período de 28 de agosto a 03 de outubro de 2014. Trata-se de um município pólo da região centro-oeste de Minas Gerais, e conta com 18 ESF e 17 serviços de assistência em saúde no nível básico.

Foi entrevistado um enfermeiro de cada ESF que tenha atendido aos critérios de inclusão: trabalhar na ESF há mais de seis meses e atender a portadores de sofrimento mental. Os enfermeiros foram escolhidos por meio de sorteio. Assim fizeram parte da amostra 13 ESF e 13 enfermeiros.

As entrevistas foram agendadas previamente com o enfermeiro responsável pela ESF. Nesse momento, explicava-se o objetivo do trabalho e questionava-o quanto à possibilidade de participação. Diante da anuência do enfermeiro em participar, a entrevista era agendada para um local e horário escolhido pelo participante.

As entrevistas tiveram um tempo médio de duração de 15 minutos e foram realizadas após esclarecimento das dúvidas e assinatura do termo de consentimento livre e esclarecido. Participaram da coleta de dados dois entrevistadores que foram previamente treinados quanto à aplicação do roteiro de entrevista e que não tinham contato prévio com os entrevistados. As entrevistas foram gravadas $\mathrm{e}$ transcritas integralmente. Os participantes concordaram em gravar seus dados e foram orientados, de acordo com a Resolução 266/12, sobre o tempo de guarda e formas de acesso aos dados ${ }^{9}$.

O roteiro de entrevista foi constituído por duas partes. Na primeira parte foram realizadas perguntas relativas aos dados demográficos como tempo de serviço na ESF, tempo de formação, presença em curso de pós-graduação e a área de atuação. A segunda parte foi constituída por duas perguntas norteadoras: Como é realizado o acolhimento aos portadores de sofrimento mental? Qual é o papel da atenção primária na rede de atenção à saúde mental?

Cada entrevista recebeu um número sequencial conforme elas foram realizadas. Os depoimentos são identificados ao final com a letra $\mathrm{E}$ (entrevista) e o número sequencial (E1, E2, E3...). Em atendimento à Resolução 466/12, o projeto foi enviado à Secretária Municipal de Saúde de Divinópolis - MG (SEMUSA) e submetido à aprovação do Comitê de Ética em Pesquisa da Fundação Educacional de Divinópolis (FUNEDI), foi aprovado sob o número do parecer 764.444.

A análise do discurso seguiu os passos descritos por Spink e Gimenes ${ }^{10}$. Inicialmente foi realizada uma leitura flutuante das transcrições e a identificação das unidades de construção das práticas discursivas. Foram então determinados os temas relevantes e as categorias analíticas que guardaram associação com os objetivos propostos. Essa etapa deu origem à elaboração do mapa de associações. Finalmente, houve a busca de sentido com base nos indicadores relacionados aos repertórios linguísticos utilizados.

\section{Resultados}

Dentre os 13 participantes, sete eram graduados entre 11 e 15 anos, 10 tinham tempo de trabalho na instituição atual (ESF) entre sete meses e cinco anos. 12 participantes cursaram pós-graduação lato sensu, em sua maioria nas áreas de ESF (sete participantes) e Saúde Pública (cinco participantes).

Foram encontradas as categorias: 1. Refletindo sobre o papel da atenção primária na assistência ao portador de sofrimento mental, 2. Acolhendo o portador de sofrimento mental.

\section{Refletindo sobre o papel da atenção primária na assistência ao portador de sofrimento mental}

O papel da atenção primária é socialmente construído e não está pronto. Conforme as mudanças sociais surgem, novos sentidos podem ser construídos pelos atores sociais conferindo alterações na prescrição desse papel. Ao refletir sobre o papel da atenção primária em relação ao atendimento dos portadores de sofrimento mental, os participantes atribuem o significado do acolhimento atrelado ao vínculo e ao suporte. 
Eu acho que é acompanhar este usuário de forma integral porque o vínculo dele é com a ESF (E3).

Acho que realmente é acolher o paciente porque a gente conhece ele muito melhor, conhece ele, conhece a família, às vezes, a gente conhece ele mais que o próprio centro de referência onde ele é tratado. Então tá aí, eu acho, a importância do acolhimento no PSF né?! (E7)

Olha a gente tem que dar o apoio que eles precisam "né"?! Tem o SERSAM [Serviço de Referência em Saúde Mental] também que faz. este acolhimento que ajuda no atendimento deles, mas nós temos que dá o suporte aqui ponta, avaliar quando tiver em crise, tentar ver o que pode ser feito pra encaminhar $e$ para onde encaminhar o que for melhor para eles. (E11)

Os enfermeiros entrevistados também indicaram a importância do acolhimento na reinserção do portador de sofrimento mental na sociedade.

Então o papel é este acolher e tentar ajudar esta pessoa a inserir na sociedade. Ajudar essa pessoa a voltar a suas atividades normais, tentar ajudar neste sentido. ( E4)

O sentido do acolhimento relacionado ao vínculo, suporte e impacto na inserção do usuário na sociedade, faz parte das práticas discursivas construídas após a Reforma Psiquiátrica. No entanto, os conceitos construídos socialmente, anteriores à Reforma psiquiátrica, não são completamente abandonados. Eles persistem nos sentidos que a sociedade e os profissionais de saúde dão ao sofrimento mental, transparecendo nas práticas discursivas em atitudes como o medo:

O medo, que a maioria chega aqui gritando, nervoso, então o funcionário fica meio imóvel diante de uma situação dessas. (E5).

Acho que as dificuldades existem, principalmente quando eles estão em crise, porque a gente fica mais receoso em tratar e temos uma dificuldade maior em tratar. (E1)

O medo é um sentimento que se faz presente e atrapalha no atendimento e no acolhimento, pois imobiliza o profissional. Ao lado de discursos ligados ao paradigma psicossocial, permanecem aqueles ligados ao modelo manicomial. $\mathrm{O}$ sentido de periculosidade relacionado ao individuo com transtornos mentais se concretiza na realidade do medo, do receio e da dificuldade em tratar, em acolher.

No entanto, o medo não está ligado somente ao paradigma manicomial, ele está na construção da relação entre o profissional e o usuário, ou seja, ele surge na história das experiências do profissional, ao atender essa clientela. Muitas vezes, o medo decorre da falta de conhecimentos para lidar com as necessidades desses indivíduos.

Ah eu acho que sim. Eu acho que falta mais conhecimento da gente "né"?! Pra toda equipe. A gente precisava ser mais capacitados.(E13).

As pessoas não são capacitadas, não estão preparadas para lidar com este tipo de paciente que as vezes são agressivos (E5).

A falta de formação e atualização em saúde mental e a necessidade de suporte profissional específico estão entre os obstáculos que trazem impacto negativo na elaboração do sentido do acolhimento em saúde mental na atenção primaria. Tal influência perpassa experiências de sofrimento vivenciadas por pacientes e enfermeiros, que se refletem em atuações percebidas como a compreensão imprecisa da forma como o acolhimento deve ser realizado, a rotulação do paciente como sendo difícil e o medo diante dos problemas de saúde mental.

\section{Acolhendo o portador de sofrimento mental}

O acolhimento está centrado na queixa apresentada pelo paciente e na respectiva forma de encaminhamento.

$O$ acolhimento é da mesma forma ne?! Alguns casos a gente tem uma psicóloga aqui que atende duas vezes por semana, então, alguns casos estes pacientes são passados para ela, os casos mais graves. Os casos que percebemos que precisam ser encaminhado para o SERSAM ou que o próprio paciente solicita ou a gente vê que tá precisando ne?! (E4)

Acolher estes pacientes e não encaminhar tudo para o SERSAM como se não fizesse parte da unidade, da nossa população. Porque tem coisas que dá pra gente resolver, não precisa mandar tudo pra lá. (E4).

As diferenças no significado do acolhimento entre os profissionais perpassam as ações de saúde desempenhadas por eles e se revelam na comparação entre o acolhimento de uma pessoa com queixas clínicas e indivíduos com transtornos mentais. Embora os profissionais reconheçam que não deve haver diferenças em relação ao acolhimento dos outros pacientes, eles admitem que elas ocorrem.

Para eu fazer o acolhimento de um paciente 'normal', que não tenha sofrimento mental, é a queixa e pronto acabou. As vezes eu vou colher preventivo de uma mulher que está passando por um sofrimento, que faz uso de medicamentos (Diazepam...), a gente acaba gastando mais tempo ouvindo, conversando com a paciente, do que para colher o preventivo em si. Então você requer uma atenção muito mais especial para esse tipo de paciente ...(E6). 
E mais você pode amenizar de alguma forma porém esse é um público muito difícil da gente trabalhar com ele é justamente porque ele é um paciente que exige mais atenção.(E6)

É possível perceber por meio dos relatos acima que o sentido atribuído pelos enfermeiros ao acolhimento desses indivíduos, está relacionado a imagens de dificuldade que embasam a percepção de que eles requerem maior atenção do que outros.

Provavelmente, na implementação do acolhimento, os pacientes com problemas de saúde mental encontram maiores barreiras para expressar suas necessidades, uma vez que recebem o rótulo de serem difíceis. $\mathrm{O}$ atendimento se torna, então, carregado de diferenças justificadas na necessidade de atender num local separado e dispensar maior tempo para a escuta.

\section{Discussão}

Os participantes percebem o acolhimento como uma ação que se integra ao vínculo e suporte. O acolhimento favorece a construção de confiança e compromisso junto aos usuários, gerando o vínculo ${ }^{11}$. Portanto, ele estimula a construção do vínculo e da confiança, possibilita maior conhecimento da clientela atendida e promove o encontro entre as pessoas, permitindo que melhores estratégias de cuidado em saúde mental possam ser construídas.

Juntos, o acolhimento e o vínculo são centrais na assistência em saúde mental na $\mathrm{ESF}^{12}$, pois ao acolher o paciente, é possível verificar e atender a outras demandas e ser referência para as pessoas com sofrimento mental ${ }^{12}$. Além desses benefícios, os participantes relataram que o acolhimento assume importante significado na reinserção do portador de sofrimento mental. Essa percepção é corroborada por estudos que concluíram que a escuta, o acolhimento e o vínculo favorecerem a reinserção social da pessoa em sofrimento psíquico, estimulam a autonomia, o exercício da cidadania e trazem o sujeito para o foco de atenção. Possibilita, ainda, desmistificar a loucura como incapacidade ${ }^{2}$, transformando a relação que a sociedade estabelece com a pessoa em sofrimento mental $^{6}$.

Entretanto, o significado da prática do acolhimento pode estar associado às ações de triagem administrativa e encaminhamentos a serviços especializados. Os entrevistados confirmam que o acolhimento da pessoa com sofrimento mental está centrado na queixa e no encaminhamento. A linha guia Atenção em Saúde Mental do Estado de Minas Gerais denuncia que, as pessoas com sofrimento mental são vistas nos serviços de saúde como pessoas difíceis de lidar e perigosas. Diante disso, os profissionais não se aproximam suficientemente desses usuários a ponto de investigar suas reais necessidades e investir no fortalecimento do vínculo.
Há, portanto, uma tendência a encaminhá-las imediatamente a um serviço de Saúde Mental especializado $^{13,14}$.

Se a decisão do encaminhamento for uma ação isolada de outros processos de trabalho, essa ação será pontual e descomprometida com processos de responsabilização e produção de vínculo. Nessa perspectiva, o principal foco do atendimento é a doença e não o sujeito e suas necessidades ${ }^{4}$.

Quando a doença se torna o principal enfoque, as ações de saúde se tornam pautadas em procedimentos técnicos, centrada na cura e na hierarquia de saberes. Esse posicionamento derivado do modelo médico hegemônico distancia o profissional do usuário e traz obstáculos à atuação profissional frente a demandas que não são físicas. Diante dessa situação, o encaminhamento a serviços especializados surge como solução, causando a desresponsabilização do profissional pela situação. $\mathrm{O}$ usuário circula entre os serviços e pode não reconhecer a nenhum deles como referência ${ }^{14}$.

Em contraste a essa realidade, a realização da prática do acolhimento concomitante a ações de saúde, é descrita na literatura. Esse caminho traz a possibilidade de um cuidado integral, interpessoal e longitudinal. A corresponsabilização pelas demandas do paciente se torna presente ${ }^{7}$. Nesse contexto, o encaminhamento para serviços especializados se torna uma ação articulada a outras, faz parte do cuidado e a equipe se mantem responsável pela situação.

$\mathrm{O}$ acolhimento realizado de maneira pontual e desarticulada de outras ações de saúde pode resultar em dificuldades percebidas por usuários e profissionais $^{7}$.

Dentre as dificuldades no atendimento a essa clientela decorrentes está o medo. No discurso do paradigma manicomial, a doença mental representa um risco, que está em maior parte ligado a uma política de normalização da cidade e da família. A pessoa pode não representar perigo potencial, mas deve ser isolada ${ }^{15}$. Desse discurso resulta o medo, que, decorrente da história da relação da sociedade com a loucura, permeia o relacionamento entre o portador de sofrimento mental, a sociedade e enfermeiro.

No discurso dos participantes, em relação aos significados do acolhimento, estão presentes o medo, o vínculo e o suporte. Isso pode indicar a coexistência de sentidos conferidos por dois modelos diferentes de atenção: o modelo psicossocial e o modelo manicomial. Essa coexistência traz empecilhos ao estabelecimento de vínculos, à pratica do acolhimento e ao cumprimento das diretrizes preconizadas pelo Ministério da Saúde relacionadas à integralidade e à resolutividade ${ }^{16}$. Dessa forma, quando questionamos os participantes sobre a função da atenção primária, prevalecem os discursos relacionados ao modelo psicossocial, mas quando 
descrevem a prática do acolhimento e suas dificuldades, os relatos se dividem entre os dois modelos.

Os conceitos trazidos pela Reforma Psiquiátrica ressignificam conteúdos arraigados ao modelo psiquiátrico tradicional. Essas transformações têm mudado gradativamente o sentir e pensar da sociedade em relação ao doente mental ${ }^{17}$. O medo é um desses conteúdos ser ressignificado.

Entretanto, o medo também pode estar relacionado à falta de capacitação e de conhecimentos necessários à atuação mais compatível com a necessidade da clientela. Os profissionais de saúde reconhecem que as pessoas com sofrimento mental devem ser acolhidas e atendidas na ESF, mas não sabem como lidar com elas e nesse sentido, o despreparo e a falta de capacitação para lidar com problemas de saúde mental são reconhecidos pelos profissionais como obstáculos à implementação do acolhimento desses usuários ${ }^{6}$.

Um estudo que verificou a avaliação dos usuários em relação ao acolhimento, observou que os usuários insatisfeitos com o acolhimento concluíram que os profissionais não estavam qualificados para recebe-los ${ }^{14}$. Portanto, essa limitação ao permear o cuidado em saúde mental é percebida por usuários e profissionais. A educação e a capacitação permanente dos profissionais são estratégias eficientes na melhora da qualidade da assistência prestada à clientela ${ }^{18}$. Por meio da capacitação, é possível a ressignificação de conteúdos e práticas na perspectiva do discurso psicossocial.

As reflexões suscitadas pela capacitação poderão ajudar na adaptação da atividade profissional ao atendimento das necessidades da clientela ${ }^{19}$. Dessa forma, comportamentos e discursos que motivaram os participantes a destinar atendimento diferenciado a usuários, poderão ser ressignificados e mudados na direção de um acolhimento que considere as necessidades de saúde de todos os usuários.

A escolha da palavra acolhimento para delimitar determinado conjunto de experiências reflete as histórias culturais e de relacionamentos que ocorreram em diferentes épocas e cenários. Dessa forma, avaliar o discurso é avaliar o cotidiano das pessoas expresso por meio de práticas discursivas ${ }^{20}$ : 63. As práticas discursivas podem mudar e exercer impacto no cotidiano do acolhimento em saúde mental. Nesse sentido, a educação em saúde pode contribuir para que os significados dos enfermeiros relacionados à saúde mental se transformem num acolhimento que considere os usuários em sua integralidade.

\section{Conclusão}

O sentido atribuído ao acolhimento de pessoas com problemas de saúde mental e, em especial, àquelas que já estão rotuladas por serem portadoras conhecidas de transtornos mentais, está relacionado à imagem de periculosidade. Esse conteúdo pertence à história da saúde mental. Esse sentido constrói a realidade que está atrás de encaminhamentos desnecessários a centros especializados, medo em atender, dificuldades na escuta e no acolhimento.

Entretanto, os discursos construídos pela Reforma Psiquiátrica e Sanitária têm adentrado esse universo e já se refletem na mudança de práticas discursivas relacionadas à importância do vínculo e do suporte. Assim, aparecem os sentidos do acolhimento relacionados ao vínculo, escuta e inserção do paciente na sociedade. Isso expressa uma alteração do significado atribuído ao acolhimento de pessoas com problemas de saúde mental. A realidade derivada desses novos sentidos transparece em atitudes de acolhimento mais humanizado.

Por enquanto, as duas práticas discursivas perpassam o cotidiano do acolhimento. No entanto, entendemos que a educação continuada e o preparo do profissional baseados no paradigma psicossocial poderão alterar gradativamente as práticas cotidianas de acolhimento, fazendo cumprir os princípios descritos pela Política Nacional de Humanização para todos os usuários.

A educação em saúde auxilia na construção de práticas discursivas que se materializam na prática assistencial, trazendo transformações. Estudos que investiguem essa questão podem estimular na materialização de discursos referentes ao modelo psicossocial na prática do acolhimento. Afinal, esta investigação tem como limite o aprofundamento acerca da maneira como os discursos se transferem para as práticas realizadas concomitantemente com o acolhimento.

Espera-se que o estudo contribua para reflexões direcionadas ao fortalecimento da humanização do cuidado em saúde mental na atenção primária.

\section{Declaração de conflitos de interesses}

Os autores do artigo afirmam que não houve nenhuma situação de conflito de interesse, tais como propostas de financiamento, emissão de pareceres, promoções ou participação em comitês consultivos ou diretivos, entre outras, que pudessem influenciar no desenvolvimento do trabalho.

\section{Referências}

1. ABRAHAMIAN,R. Uma experiência de psicoterapia de grupo dentro da estratégia saúde da família. Revbrasmedfam comunidade,v.6, n. 21, p. 271-274,2011. 
2. SIMÕES, W.M.B.; MOREIRA, M.S.A. importância dos atributos: acolhimento, vinculo e longitudinalidade na construção da função de referencia em saúde mental na atenção primária à saúde. Rev. Enfermagem Revista, v.16, n. 3, p. 223-239, set/dez, 2013.

3. Brasil. Ministério da Saúde. Secretaria de Atenção à Saúde. Departamento de Atenção Básica. Cadernos de Atenção Básica n 34. Brasília: Ministério da Saúde; 2013.

4. FARIA, M.L.C.; GUERRINI, I.A. Limitações do paradigma científico hegemônico no acolhimento ao sofrimento psíquico na atenção básica. InterfaceComunic, Saúde, Educ.,;v. 16, n. 42, p. 779-792, jul/set, 2012.

5. MIELKE, F.B.; OLSCHOWSKY,A. Ações de saúde mental na estratégia saúde da família e as tecnologias em saúde. Esc. Anna Nery (imp), v. 15, n. 4, p. 762-768, outdez, 2011.

6.ANJOS, M.A.; CARVALHO, P.A.L.; SENA, E.L.S.; RIBEIRO, R.M.C. acolhimento da pessoa em sofrimento mental na atenção básica para além do encaminhamento. Cadernos Brasileiros de Saúde Menal, Florianóplis, v 7, n 16, p 27-40, 2015.

7.COUTINHO, L.R.P.; BARBIERI, A.R.; SANTOS, M.L.M. Acolhimento na atenção primária à saúde: revisão integrativa. Saúde Debate, Rio de Janeiro, v 39, n 105, p 514-524, abr-jun, 2015.

8.CASTAÑON, G.A. Construcionismo social: uma crítica epistemológica. Temas em psicologia SBP. 2004, 12 (1): 67-81.

9.BRASIL. Conselho Nacional de Saúde. Diretrizes e Normas Regulamentadoras de Pesquisas Envolvendo Seres Humanos. Resolução 466/12, Brasília, 2012.Disponível em: http://conselho.saude.gov.br/resolucoes/2012/Reso466.pdf. Acesso em 11 nov 2015

10. SPINK, MJ; GIMENES, M.G. Práticas discursivas e produção de sentido: apontamentos metodológicos para analise de discursos sobre a saúde e a doença. Saúde e sociedade, v. 3, n. 2, p. 149-171, 1994.

11. FERREIRA, M.S.L.S.M.; PENQUES, R.M.V.B. Acolhimento na percepção dos enfermeiros da atenção primária à saúde. Arquichan, Chía, v 14, n. 2, p. 216-225, junio, 2014.
12. MAGALHÃES, V.C.; PINHO, L.B.; LACCHINI, A.J.B.; SCHNEIDER, J.F.; OLSCHOWSKY,A. Ações de saúde mental desenvolvidas por profissionais de saúde no contexto da atenção básica. R. pesq.: cuid. fundam.,v.4, n. 4, p. 3105-3117, out./dez, 2012.

13. Minas Gerais. Secretaria de Estado de Saúde. Linha Guia da Saúde Mental. Belo Horizonte, 2006. 238 p. Disponível em: http://www.saude.mg.gov.br/publicacoes/linha-guia/linhasguia. Acesso em: 06 set 2015.

14. FRATESCHI, M.S.; CARDOSO, C.L. Saúde menal na atenção primária à saúde: avaliação sob a otica dos usuários. Physis Revista de Saúde Pública, Rio de Janeiro, v 24, n 2, p 545-565, 2014.

15. MALAMUT, B.S.; MODENA, C.M.; PASSOS, I.C.F.Violência e poder no discurso psiquiátrico: da exclusão às subjetivações normativas. PsicolSoc, Florianópolis, v. 23, n. especial, p. 53-62, 2011.

16. ARAUJO, A.K.; TANAKA, O.U. Avaliação do processo de acolhimento em saúde mental na região centrooeste do município de São Paulo. Interface- Comunic, Saúde, Educ., v. 16, n. 43, p. 917-928, 2012.

17. PINHO, L.B.; KANTORSKI, L.P.; OLSCHOWSKY, A.; SCHNEIDER, J.F.; LANCCHINI, J.B.Ideologia e saúde mental: análise do discurso do trabalhador no campo psicossocial. Texto Contexto Enferm, Florianópolis, v. 23, n. 1, p. 65-73, jan/mar, 2014.

18. CORREIA, V.R.; BARROS, S.; COLVERO, L.A. Saúde mental na atenção básica: prática da equipe de saúde da família. RevEscEnferm USP [Internet], v. 45, n. 6, p. 1501-1506, 2011. Disponível em<http://www.scielo.br/scielo.php?script=sci_arttext\&pid $=$ S0080 $-62342011000600032 \& \operatorname{lng}=$ en $\& n r m=i s o>$. Acesso em 30 jun. 2016.

19. MACEDO, C.A.; TEIXEIRA, E.R.; DAHER, D.V. Possibilidades e limites do acolhimento na percepção de usuários. Rev. enferm. UERJ, Rio de Janeiro, v. 19, n. 3, p. 457-462, jul/set, 2011.

20. GUANAES-LORENZI, C.; MOSCHETA, M.S.; CORRADI-WEBSTER, C.M.; SOUZA, L.V.Construcionismo social: discurso, prática e produção do conhecimento. Rio de Janeiro: Instituto Noos, 2014. 